\title{
Gesta de Malvinas - 1982: un capítulo más a la Historia de la Medicina
}

\author{
Gesta de Malvinas - 1982: one more chapter in the \\ History of Medicine
}

Melisa A. Gutiérrez Quiroga', Sergio Verbanaz², Pablo Young²

\section{RESUMEN}

Los ataques de las fuerzas británicas pusieron a prueba a los equipos sanitarios militares de las tres fuerzas armadas durante 74 días. La evacuación desde el campo de batalla hacia los diversos centros de complejidad fue realizada sin distinción de rango ni género, una tarea que merece ser recordada, no solo por el coraje y el compromiso de los agentes de salud, sino también por la eficacia y entereza que tuvieron en trabajar por la vida en un contexto en el que solo cabía la muerte. Este documento, basado en fuentes orales y escritas, relata la eficaz sistematización de la salud en las Islas Malvinas durante el conflicto, hace una especial mención a las mujeres que tuvieron una destacada tarea brindando su profesionalismo y empatía, y por último describe una afección olvidada que sorprendió a la mayoría de los médicos: el pie de trinchera.

Palabras clave: Malvinas, medicina, guerra, evacuación, mujeres, pie de trinchera.

\begin{abstract}
The attacks of the British armed forces tested the military health teams of the three armed forces for 74 days. The evacuation from the battlefield to the various centers of complexity was done without distinction of rank or gender, a task that deserves to be remembered, not only because of the courage and commitment shown by health agents, but also for the efficiency and integrity that they had in working for life in a context where there was only death. This document, based on oral and written resources, recounts the effective systematization of health in Falkland Islands during the conflict, makes special mention of women who has an outstanding task offering their professionalism and empathy and finally describes a forgotten condition that surprised most doctors: the trench foot
\end{abstract}

Keywords: Malvinas, medicine, war, evacuation, women, trench foot.

\section{INTRODUCCIÓN}

\section{"Tu vocación reside abi, donde se cruzan tu talento y las necesidades del mundo." Aristóteles}

La guerra oficialmente comenzó los primeros días de mayo con los ataques aéreos británicos, fundamentalmente nocturnos, sobre Puerto Argentino y Darwin. La desproporción entre las tecnologías de guerra británicas y argentinas produjo un incesante número de bajas y heridos. La rapidez de la atención médica se vio severamente afectada por la oscuridad, la geografía y el inclemente frío austral.

Es encomiable la vocación de los médicos, odontólogos,

\footnotetext{
I. Alumna de cuarto año, Cátedra de Historia de la Medicina,

2. Cátedra de Historia de la Medicina, Universidad Católica Argentina.
}

Correspondencia: Dr. Pablo Young. Servicio de Clínica Médica, Hospital Británico. Perdriel 74, CI280AEB CABA, Rep.Argentina. Tel 54II 43096400. Fax 54II 43043393.Email: pabloyoung2003@ yahoo.com.ar

Los autores declaran no poseer conflictos de intereses.

Recibido: 14/06/2018 | Aceptado: 01/7/2018 bioquímicos y farmacéuticos que trabajaron ardua y profesionalmente en el frente de batalla. Este documento tiene como objetivo relatar cómo se organizaron las fuerzas armadas para proveer la mejor atención médica posible y cuál fue la logística empleada, en un contexto poco favorable, hacer una mención especial a las mujeres veteranas de guerra y al pie de trinchera, que fue la causa de ingreso a la guardia más frecuente durante el conflicto.

\section{BREVE DESARROLLO DEL CONFLICTO}

El conflicto bélico en las Islas Malvinas comenzó a fines de marzo de 1982. El paso inicial fue el desembarco de un grupo de obreros argentinos que tenían la misión de instalar factorías balleneras. Esto representó para el gobierno británico, una invasión. La escalada se agravó cuando el gobierno argentino decidió enviar catorce efectivos, con el fin de proteger la integridad física de los obreros, y dar inicio a la recuperación de las islas mediante la conocida Operación Rosario ${ }^{1}$. Dicha maniobra se produjo el 2 de abril de 1982, día que en que recordamos la Soberanía Argentina sobre las Islas Malvinas. Este hecho provocó la ruptura de relaciones con el gobierno británico, dando lugar al conflicto bélico más importante en esta parte del mundo durante el siglo XX.

La Gesta de Malvinas se podría dividir en dos grandes períodos $^{2}$. El primero se desarrolló desde el 1 al 27 de mayo 


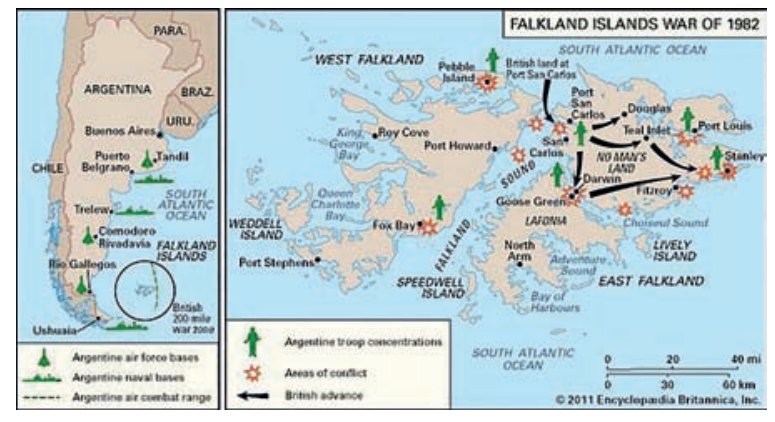

Figura I. Mapa de las Islas Malvinas, junto a las batallas mencionadas en el texto. http://fdra-malvinas.blogspot.com.ar/20 16/04/las-locaciones-y-los-lugares-de-batalla.html (consultado 07/02/20/8).

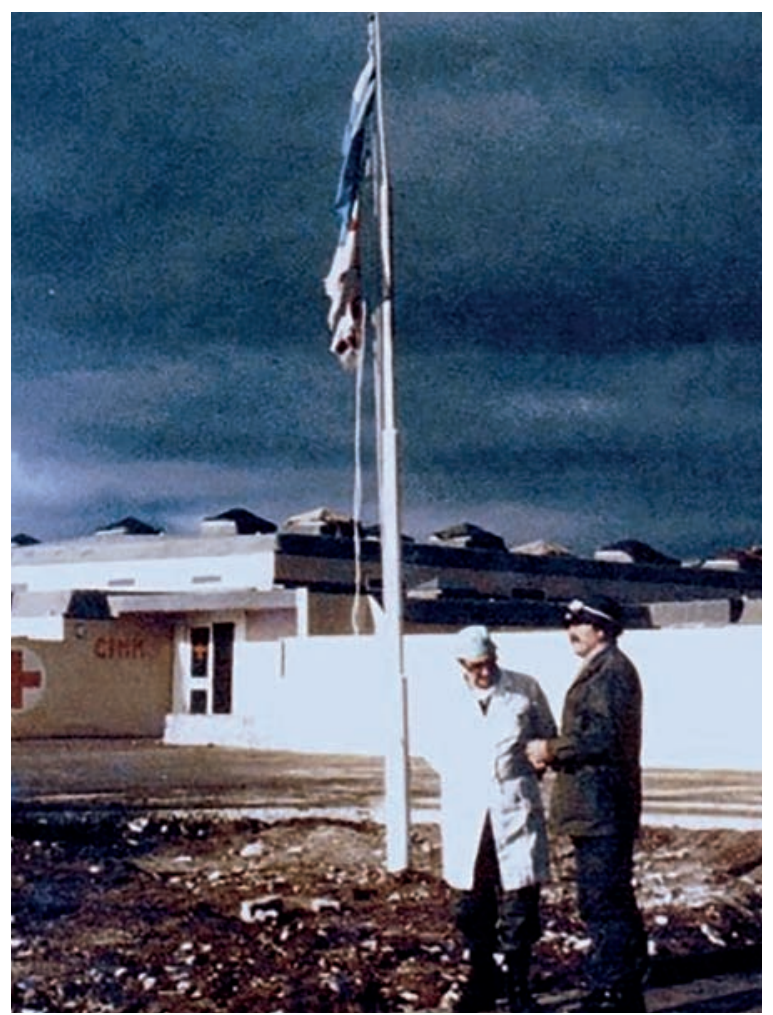

Figura 3. El Dr. Mayor Espiniella junto con el Dr. Mayor Ceballos en las afueras del CIMM. https://www.taringa.net/posts/info//22 I /2 I / La-Guerra-de-MalvinasPost-Merecido-Parte-15.html (consultado 07/02/20/8).

caracterizado por los constantes bombardeos aéreos ingleses sobre las islas, produciendo un continuo y arduo desgaste de las tropas argentinas. Como consecuencia el ejército inglés tomó como cabecera de playa, San Carlos (Isla Soledad) entre los días 20 y 21 de Mayo 1 .

El segundo período se inició con el Combate de Goose Green-Darwin los días 27 y 28 de Mayo, y se extendió hasta el día 11 de Junio, día en el que se produjo el Combate en Puerto Argentino, que finalizó con la rendición argentina (Figura 1) ${ }^{1}$.

\section{DISTRIBUCIÓN DE LA MEDICINA EN COMBATE}

La medicina fue ejercida por el Servicio de Sanidad que forma parte de los Servicios de Apoyo de Combate

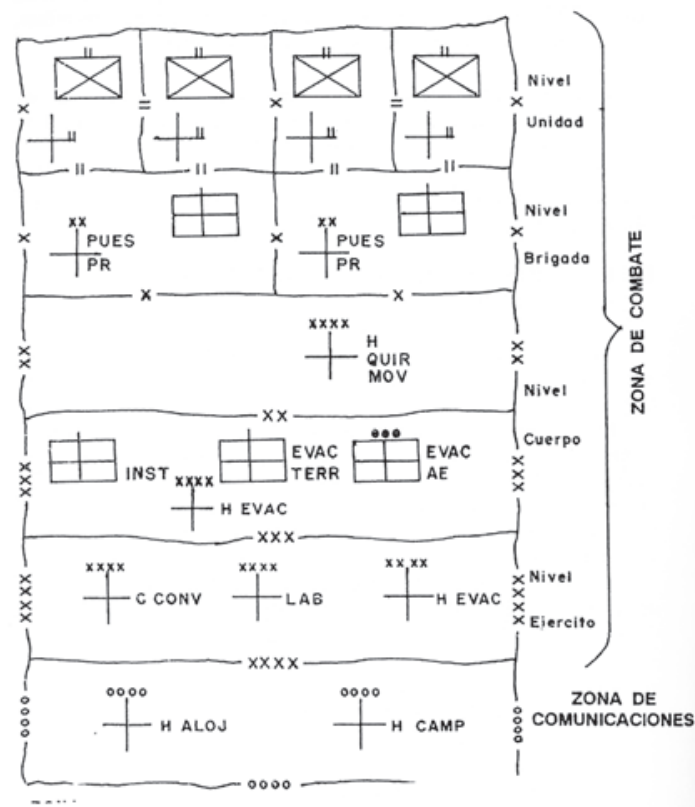

Figura 2. Esquema del despliegue de la medicina en el teatro de operaciones². PUES PR: puesto principal de socorro. H QUIR MOV: Hospital Quirúrgico Móvil. EVACTERR: Evacuación Terrestre. EVAC AE: Evacuación aérea. H EVAC: Hospital de Evacuación. H ALOJ: Hospital Alojamiento. H CAMP: Hospital de campaña (CIMM).

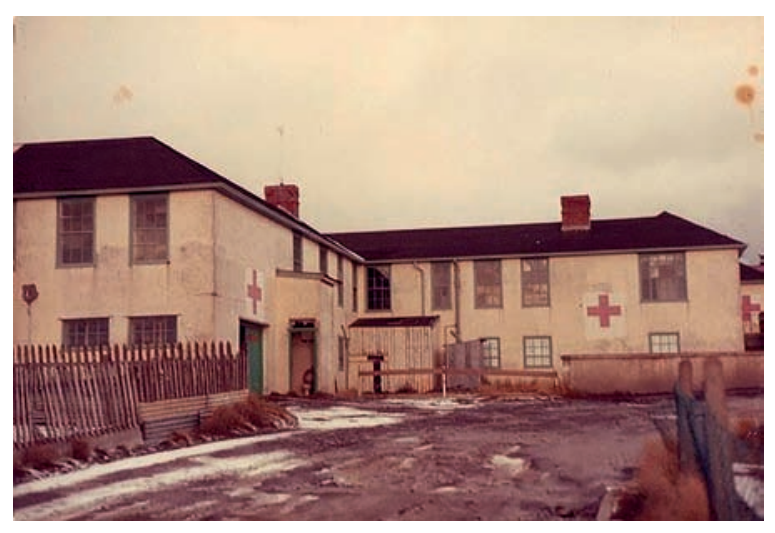

Figura 4. Centro Interfuerzas Médico Malvinas - Hospital Militar Puerto Argentino. http://londonfirejournal.blogspot.com.ar/2014/09/hospital-fire-1 984.html (consultado 07/02/2018).

del Ejército. Este Servicio se organizó y ejecutó tareas basándose en "Conducción del Servicio de Sanidad en el Teatro de Operaciones” de 1966, donde se detallan las limitaciones, operaciones y acciones ante distintas situaciones tácticas, así como las funciones del servicio: ejecución de la medicina preventiva, atención de los pacientes, hospitalización, evacuación de enfermos y heridos, abastecimiento de sanidad, mantenimiento de sanidad, apoyo de registro necrológico, inteligencia en sanidad ${ }^{2}$.

Tanto en la Isla Soledad como en Isla Gran Malvina, el Ejército Argentino se organizó en dos zonas principales, la de combate y la de comunicaciones. La primera se encontraba dividida en 3 niveles, dentro de las cuales el Servicio de Sanidad apoyaba de manera diferente (Figura 2): 


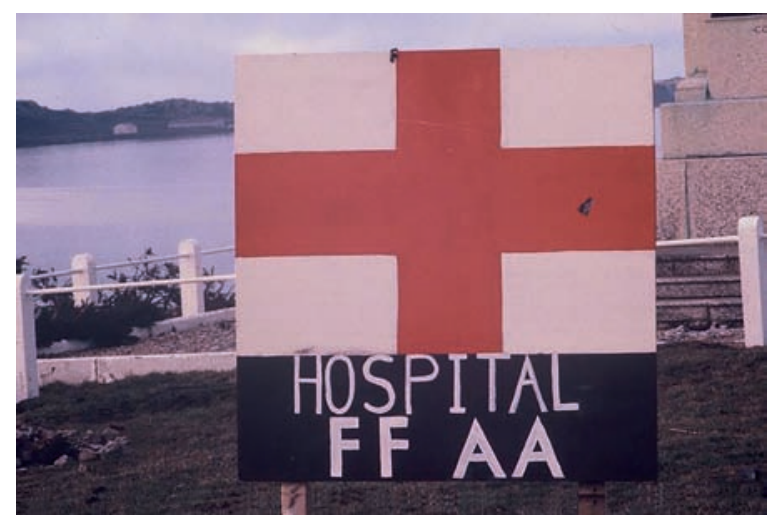

Figura 5. Logo del CIMM. https://www.taringa.net/posts/info// 22 I / 2 I I/La-Guerra-de-Malvinas-Post-Merecido-Parte-I 5.html (consultado 07/02/20/8).

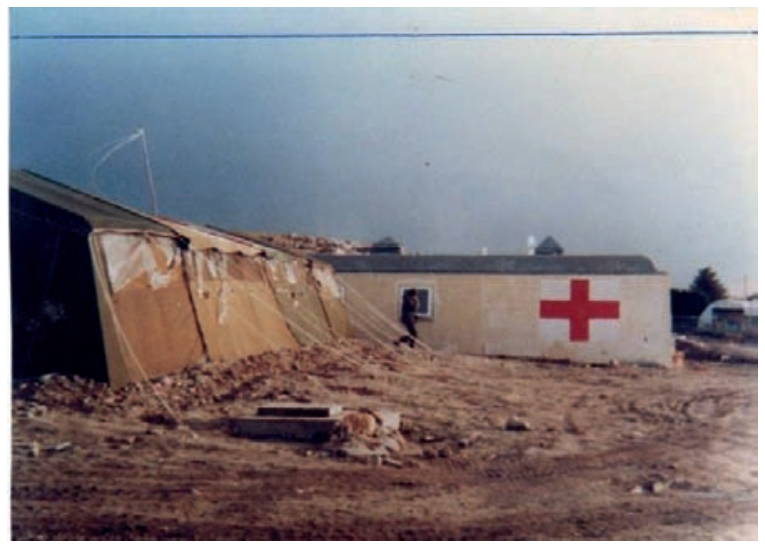

Figura 7. Carpa necrológica vecina al CIMM. https://www.taringa.net/posts/ info// 22 I I 2 I / /La-Guerra-de-Malvinas-Post-Merecido-Parte-I5.html (consultado $07 / 02 / 2018)$.

- A Nivel de Unidad, la Sección de Sanidad estaba compuesta por diversos grupos de hombres que cumplían funciones asignadas, tales como: grupo comando de 5 hombres, grupo de evacuaciones de 10 camilleros, grupo de 10 enfermeros y grupo de 10 hombres encargados del registro necrológico; todos ellos a cargo de un oficial médico, quien ejercía su función en un Puesto de Socorro.

- A Nivel de Brigada, el apoyo sanitario era suministrado por la Compañía de Sanidad del Batallón Logístico, compuesta por grupos comando, de evacuaciones, de mantenimiento y abastecimiento, $\mathrm{y}$ de registro necrológico, así como por 6 médicos, 1 odontólogo, 1 bioquímico y 1 farmacéutico. Los profesionales ejercían en dos Puestos Principales de Socorro.

- A Nivel Cuerpo del Ejército, aquí eran trasladados los heridos por Hospitales Quirúrgicos Móviles hacia los Hospitales de Evacuación, que se encontraban en dicho nivel, donde también se determinaba la evacuación por vía terrestre o aeronáutica, dependiendo de la gravedad del paciente.

Los Puestos de Socorro merecen una destacada mención, ya que tanto los profesionales como los combatientes realizaban en conjunto el triage de los heridos, determinando quiénes estaban aptos para la evacuación

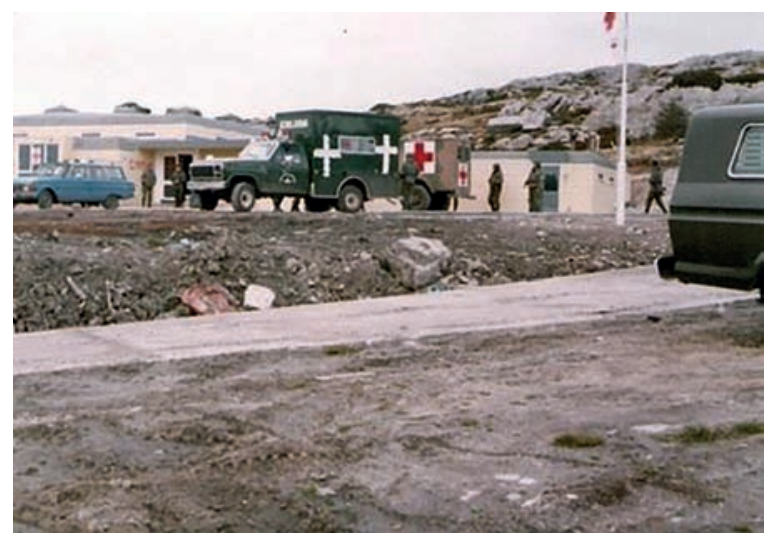

Figura 6. EI CIMM de fondo, la pista para ambulancias y las mismas estacionadas. http://www.3040 100.com.ar/wp-content/uploads/2013/04/40-p.jpg (consultado $07 / 02 / 2018)$.

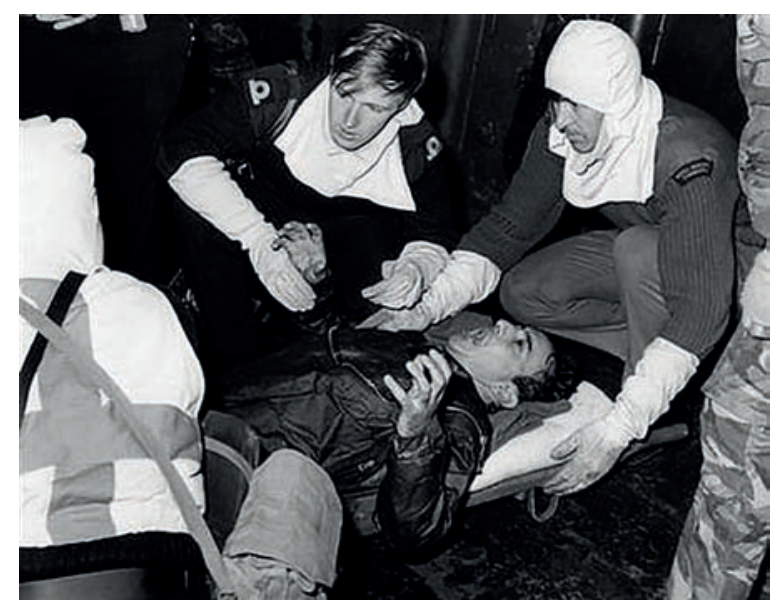

Figura 8. Teniente Ricardo "Tom" Lucero atendido por ingleses a bordo del Hospital Militar Fearless. https://www.zona-militar.com/foros/threads/im\%C3\%AI genesdel-conflicto-de-malvinas-fotos.258/page- 248 (consultado 07/02/20/8).

al siguiente nivel de complejidad; cada Regimiento de Infantería contaba con un puesto de socorro. La táctica de los ingleses de bombardear en forma incesante de noche y por vía aérea tornó muy dificultoso tanto el triage como la evacuación, agravada esta última por la geografía del lugar, ya que la única vía de evacuación era la terrestre, o sea a pie. De esta manera, aquellos heridos que, a pesar de ser clasificados como emergencias, recibían atención luego de casi 6 u 8 horas de traslado, en su gran mayoría llegaban sin vida al Hospital Militar de Puerto Argentino (HMPA). Sin embargo, algunos fueron salvados gracias a la vocación de los profesionales como al coraje de sus pares (Figura 3). Ya en la zona de comunicaciones, el apoyo sanitario era provisto por los Hospitales Generales y de Alojamiento.

\section{HOSPITAL MILITAR PUERTO ARGENTINO - CENTRO INTERFUERZAS MÉDICO MALVINAS}

Los hombres que llevaron a cabo la Operación Rosario, pertenecientes a las distintas fuerzas argentinas, se encontraban sin atención médica hospitalaria. Por ello, 


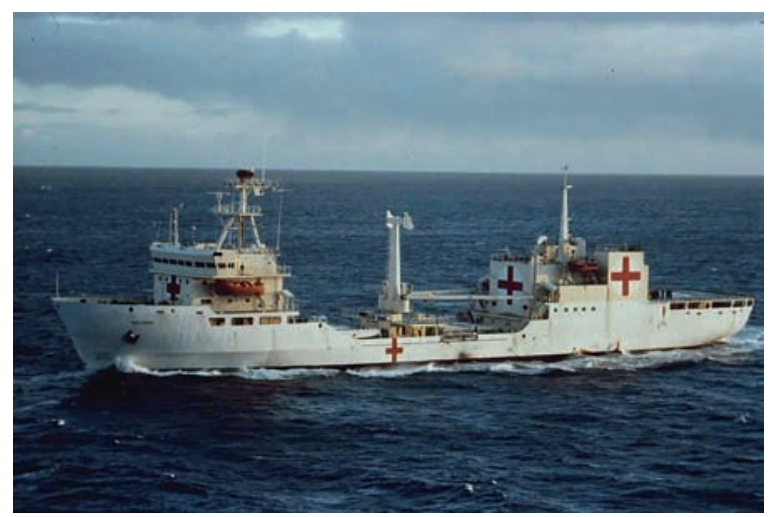

Figura 9. Buque Hospital ARA Bahía Paraíso ubicado en la "Red Cross Box" http:// www.histarmar.com.ar/Armada\%20Argentina/Buques / 900a / 970/BPolares/BPBParaiso.htm (consultado 07/02/2018).

el 5 de abril el Comandante Terrestre le ordenó al Director del Hospital Militar de Comodoro Rivadavia el traslado de personal militar, dirigido por el Dr. Mayor Enrique Ceballos, a Puerto Argentino con el fin de instalar un hospital. El 7 de abril, arribó un grupo perteneciente a la Fuerza Aérea, con el fin de brindar apoyo; este grupo estaba compuesto por el Mayor Fernando Espiniella (Jefe del Equipo Médico), los Mayores Juan Martin (Equipo Médico) y Roberto Sturtecky (Equipo de Odontología), 1er Teniente Alberto Fernández (Equipo Bioquímica) y los enfermeros Carlos Ortiz y Daniel Quiroga (Figura 4) ${ }^{3}$.

Debido a que el personal de la atención sanitaria formaba parte de las tres fuerzas armadas (ejército, naval y aérea), el HMPA, luego de su instalación entre los días 10 y 11 de abril, pasó a denominarse Centro Interfuerzas Médico Malvinas (CIMM) (Figuras 5, 6 y 7$)^{4}$.

El personal sanitario, al día 3 de junio, estaba compuesto por distintos profesionales médicos de las tres fuerzas: clínicos, anestesistas, cirujanos generales, vasculares, plásticos, traumatólogos, neurocirujanos, urólogos, bioquímicos, odontólogos, farmacéuticos y enfermeros ${ }^{2}$.

El CIMM, a mediados del mes de abril, contaba con: sala de guardia, de recepción y clasificación de heridos, de internación (2), de operaciones, de yesos, de radiografías, laboratorio, farmacia, depósito de cadáveres (Figura 7), playas de estacionamiento para ambulancias, y un helipuerto ubicado a 50 metros del establecimiento 5 .

\section{ATENCIÓN MÉDICA EN EL CIMM}

Es sabido que la atención sanitaria en un contexto de guerra es abismalmente diferente de una guardia o consultorios externos en un hospital urbano, debiendo tenerse en cuenta la logística en el traslado del paciente desde el lugar donde se produjo la lesión hasta el centro de atención, la rápida y eficiente clasificación del mismo una vez arribado, la rápida y adecuada evacuación a otro centro en caso de que sea necesario además de todo lo que respecta a la actividad médica específica, sin olvi-

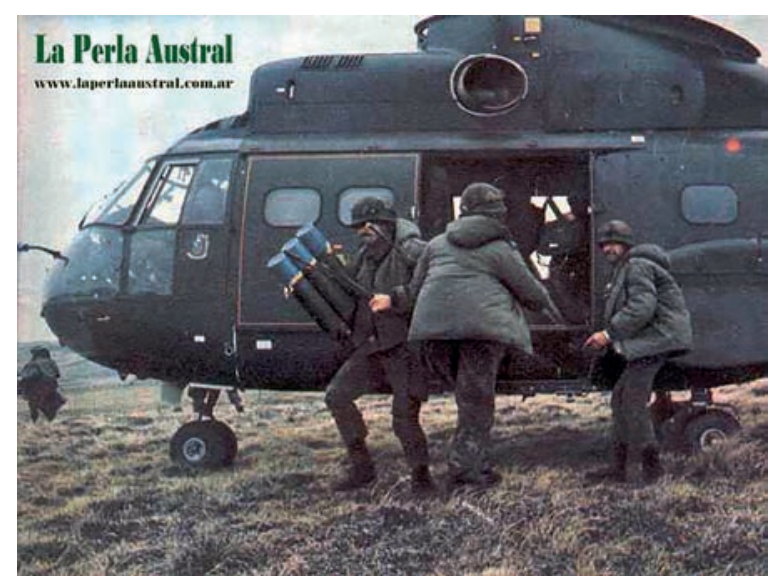

Figura I0. Puma SA-330. http://fdra-malvinas.blogspot.com.ar/2016//0/helicoptero-medio-aerospatiale-sa-330.html (consultado 07/02/20I8).

Tabla I. Motivos de internación registrados en el HMPA.

\begin{tabular}{|l|c|c|}
\hline Afecciones relacionadas con el combate & Número & $\%$ \\
\hline Heridos por arma de guerra & 361 & 18,14 \\
\hline Pie de trinchera & 173 & 8,69 \\
\hline Afecciones no relacionadas con el combate & & 73,17 \\
\hline Apendicitis aguda & 5 & \\
\hline Gastroenteritis & 156 & \\
\hline Uñas encarnadas infectadas & 13 & \\
\hline Infecciones de piel & 107 & \\
\hline Lumbociatalgias & 60 & \\
\hline Neumopatías & 56 & \\
\hline Síndromes gripales & 45 & \\
\hline Artralgias & 44 & \\
\hline Anginas & 43 & \\
\hline Micosis de piel & 42 & \\
\hline Eritema pernio & 40 & \\
\hline Quemaduras & 34 & \\
\hline Otitis media supurada & 24 & \\
\hline Síndrome de estrés postraumático & 21 & \\
\hline Infecciones urinarias & 19 & \\
\hline Desnutrición & 14 & \\
\hline Hepatitis endémicas & & \\
\hline Total & & \\
\hline
\end{tabular}

dar la táctica de ataque por parte del enemigo, ya que de ella dependerá todo lo anteriormente mencionado ${ }^{2-4}$.

El CIMM, ubicado en Isla Soledad, debió hacer frente a un impedimento en la evacuación por vía marítima hacia el continente debido a que fuerzas navales inglesas bloqueaban dichas rutas desde el mes de abril. Esto tuvo clara repercusión en el resultado final de los pacientes al afectar el "período de oro" de los mismos.

Una vez arribado al CIMM, se realizaba el triage determinando la prioridad de tratamiento o evacuación. Esta tarea se realizó teniendo en cuenta, en primer lugar, las circunstancias generales del paciente, es decir, si requería inmediatamente RCP o cirugía de emergencia, si ambas no eran necesarias y el paciente podía esperar, o si se tenía certeza de que se iban a llevar a cabo sin éxito. En segundo lugar, se tuvieron en cuenta las circunstancias particulares del CIMM, como la disponibilidad de mesas qui- 


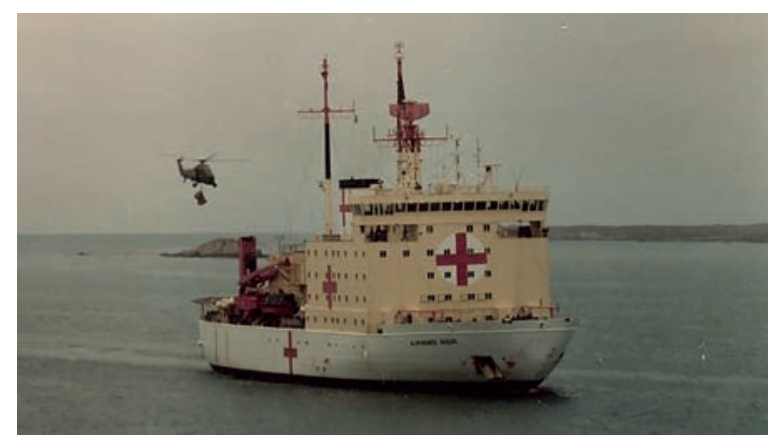

Figura II. Helicóptero arribando al Buque Hospital Almirante Irízar. http://www. irizar.org/malvinas-mujeres-inicio.html (consultado 07/02/20/8).

Tabla 2. Las técnicas quirúrgicas según el órgano lesionado.

\begin{tabular}{|l|l|}
\hline Lesión en órgano & Técnica quirúrgica empleada \\
\hline Duodeno & Exclusión pilórica o "diverticulización" \\
\hline $\begin{array}{l}\text { Intestino delgado y es- } \\
\text { tómago }\end{array}$ & Suturas o extirpación parcial \\
\hline Colon & Colostomía \\
\hline Recto subperitoneal & $\begin{array}{l}\text { Colostomía + drenaje del espacio pel- } \\
\text { virrectal }\end{array}$ \\
\hline Bazo & Esplenectomía \\
\hline Hígado & Ligadura de hilio hepático \\
\hline Páncreas & $\begin{array}{l}\text { Resección distal de la lesión. La duode- } \\
\text { nopancreatectomía se realizó ante com- } \\
\text { promiso de este órgano y duodeno. }\end{array}$ \\
\hline Aparato urinario & $\begin{array}{l}\text { Vías urinarias se repararon endosocó- } \\
\text { picamente, en cambio si el riñón estaba } \\
\text { comprometido se extirpaba, con la pre- } \\
\text { via evaluación funcional del riñón con- } \\
\text { tralateral. }\end{array}$ \\
\hline
\end{tabular}

rúrgicas, ambulancias o helicópteros para la evacuación. Los encargados del triage fueron los anestesistas.

En cuanto a los heridos (Tabla 1), el 70\% de los internados ingresaron por causas parcialmente ajenas al combate. De los 361 heridos por arma de guerra, el $82 \%$ pertenecían al ejército. El tipo de arma de guerra causante de las lesiones fue, en el 70\% de los casos, esquirlas de munición de artillería. Estas generaron lesiones en miembros superiores responsables de amputaciones de brazo, mano y dedos de la mano, y en miembros inferiores produjeron fracturas expuestas de fémur, tibia y peroné y huesos del pie. En el 30\% de los casos restantes, las lesiones fueron causadas por explosiones, pie de mina y mano de granada.

El tratamiento de los heridos comprendió tratamiento quirúrgico, teniendo en cuenta el sitio de la lesión, además de suministrar antibióticos (desvitalización de tejidos que generan tanto las esquirlas como las explosiones), y resucitación, que comprende: permeabilizar la vía aérea, colocar vías para hidratar y administrar sustancias vasoactivas para la estabilización circulatoria, detener hemorragia si las hubiera, drenaje percutáneo de neumotórax o taponamiento cardíaco y evaluación neurológica para descartar lesiones en los cuerpos vertebrales, las cuales agravarían el cuadro si el paciente era movilizado².

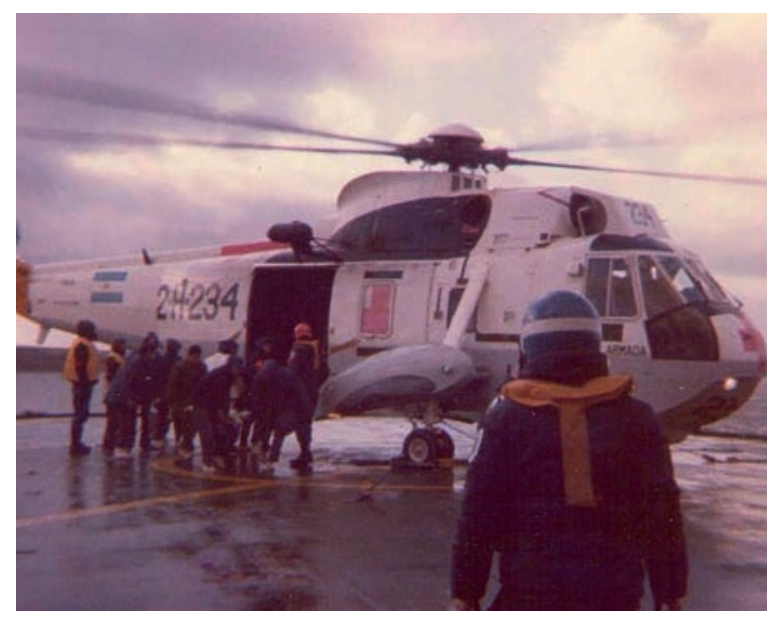

Figura I2. Sea King 234 evacuando pacientes a bordo del Irizar. http://www.irizar.org/RHAIMLV.html (consultado 07/02/20/8).

El abastecimiento, administración y distribución de unidades de sangre en el CIMM estuvo a cargo de un bioquímico, al no contar con médico hemoterapeuta. Las mismas se obtuvieron por vía marítima (buques hospitales) y vía aérea, provenientes de la Dirección Sanidad de la Fuerza Aérea, entidad que recibió unidades desde los Hospitales Penna y Churruca, donde se produjeron donaciones de los efectivos pertenecientes a dicha fuerza que se quedaron en continente y de la sociedad argentina en general 5 .

El tratamiento empleado en los heridos con lesiones en miembros incluyó, en primer lugar, el desbridamiento de los tejidos desvitalizados junto con la determinación clínica de la viabilidad muscular del miembro afectado, observando la presencia de sangrado o no cuando se lo seccionara, si tenía un aspecto cianótico, y la contracción del mismo ante un estímulo mecánico. El desbridamiento se llevó a cabo principalmente en el Hospital de Campaña de Puerto Argentino, y bajo determinados principios: incisiones amplias en dirección al eje de la extremidad, extirpación de todos los tejidos necrosados, amplio desbridamiento, abundante irrigación con solución fisiológica, dejando la herida abierta, cierre de la herida a los 4 o 5 días, luego de comprobar la limpieza de la misma².

Debido a la desmesurada afluencia de heridos que ingresaban requiriendo atención urgente, estos pacientes eran derivados al continente para efectuar el cierre de heridas, paso que, en varias ocasiones, se postergaba debido a las dificultades en las evacuaciones antes puntualizadas.

Además del desbridamiento, también se extrajeron cuerpos extraños, tales como balas o esquirlas, y se evaluó, tanto clínicamente como con exámenes complementarios (eco-Doppler y/o angiografía), el aparato circulatorio para evidenciar lesiones vasculares.

En el caso de que el paciente ingresara por fracturas expuestas, el tratamiento se basó en la inmovilidad del miembro por tutores externos. 


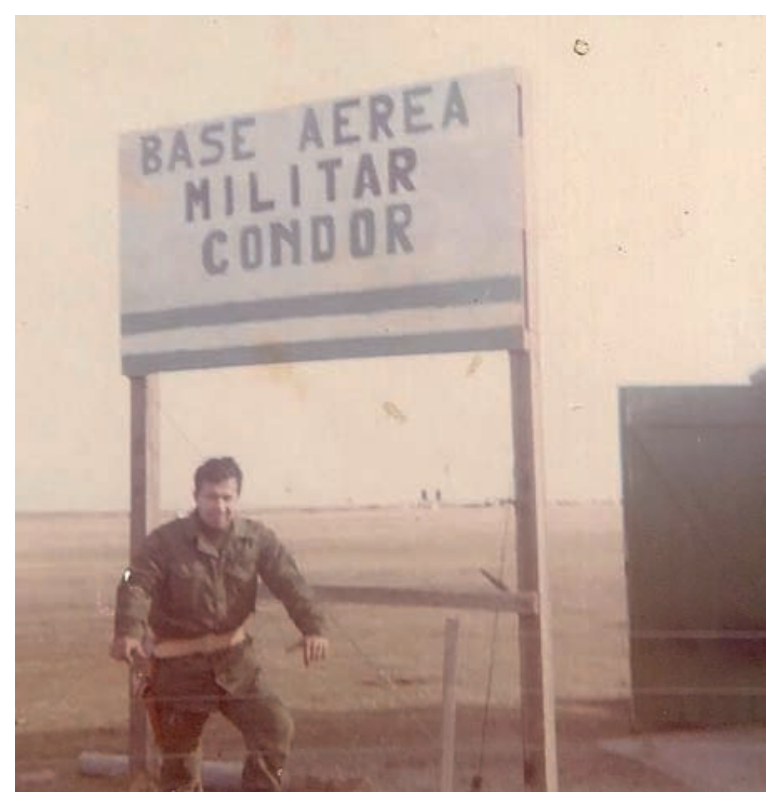

Figura I3. Soldado en la BAM Cóndor. http://extension.unicen.edu.ar/familia/galeria-de-fotos-de-malvinas/bam-condorl (consultado 07/02/20/8)

El primer accionar ante las heridas en abdomen fue la laparotomía exploradora, tanto si se hallaban signos clínicos positivos (signos de defensa, dolor a la descompresión, distensión o aire subdiafragmático) o no, o en caso que se observaran orificios de ingreso y/o salida de los proyectiles.

En el paciente que ingresaba se adoptaba la siguiente secuencia:

- Preparación del campo quirúrgico.

- Las incisiones que se aplicaron fueron medianas o paramedianas.

- Una vez abierto el peritoneo, se efectuaba en orden de prioridades:

- Evacuación de sangre y coágulos.

- Control de las hemorragias.

- Control de las lesiones de vísceras huecas.

- Reparación de otras vísceras intraabdominales.

- Exploración del retroperitoneo, si está indicado.

- Colocación de drenajes.

- Cierre del abdomen.

Las lesiones en los respectivos órganos fueron tratadas con técnicas quirúrgicas adecuadas (Tabla 2) 2 .

El 85\% de las heridas de tórax se controlaron y solucionaron con la indicación de drenaje. Las indicaciones para toracotomía fueron:

- Heridas del corazón o grandes vasos, o su sospecha.

- Hemopericardio con taponamiento, o su sospecha.

- Lesión de esófago, o su sospecha.

- Hemorragia continua por el tubo de drenaje torácico o gran pérdida de aire por el mismo².

Por último, las heridas craneoencefálicas fueron indicación de craneotomía exploradora.

Ante las heridas maxilofaciales fue indispensable la permeabilización de la vía aérea, generalmente, con

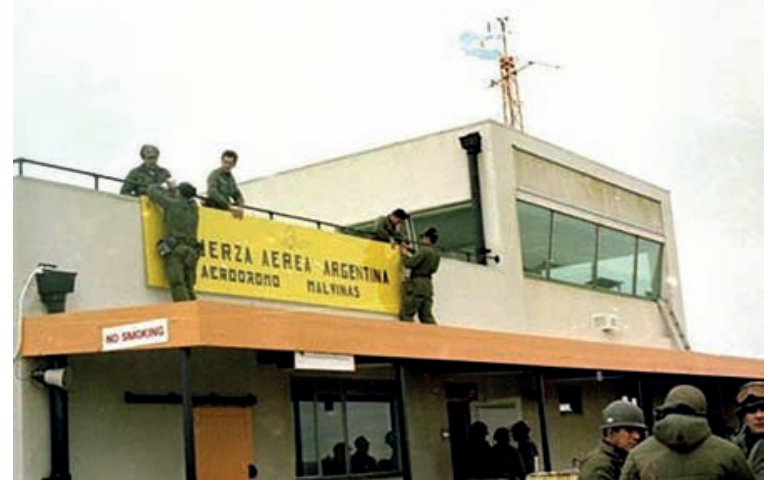

Figura 14. Soldados ultimando detalles en la BAM Puerto Argentino. http:// www.3040 I00.com.ar/los-soldados-heroes-de-malvinas/ (consultado 07/08/20/8).

una traqueostomía. Si además el paciente presentaba hemorragia, se recurría a la ligadura de la carótida externa.

Las heridas del cuello siempre obligaron la exploración de la zona.

\section{LOS BUQUES HOSPITAL:ARA BAHÍA PARAÍSO Y ARA ALMIRANTE IRÍZAR}

Los buques hospitales británicos y argentinos tuvieron, en su instalación y adecuación, los lineamientos de la Convención de Ginebra del 12 de agosto de 1949².

Los buques hospitales se instalaron en un radio de seguridad de veinte millas de diámetro ubicado al norte de las islas, en una zona que se denominó "Red Cross Box". Allí trabajaron conjunta y arduamente durante los 74 días que duró el conflicto, para brindar la mejor atención médica a sus heridos. El Comité Internacional de la Cruz Roja veló para que esto se llevara a cabo de manera eficaz (Figura 8) $)^{4}$.

\section{Buque ARA Bahía Paraíso (Figura 9)}

Previo a los primeros días del mes de abril de 1982, este buque cumplía funciones de expedición y transporte. El 13 de abril comenzaron las reformas edilicias del buque como buque hospital, que duraron aproximadamente dos meses; al mismo tiempo arribaron vía aérea suministros indispensables, tales como tubos de oxígeno, medicamentos, instrumental de cirugía, equipos, etc. Una vez adaptado, contaba con: dos salas de internación con 120 camas cada una, 4 quirófanos, laboratorio de análisis clínicos y hemoterapia, dos salas de rayos, sala de terapia intensiva, pista para helicópteros, un helicóptero Puma SA-330 (Figura 10) con capacidad de transportar 8 camillas, y dos centros de recepción y clasificación de heridos (uno recibía por vía marítima, y otro por vía aérea $)^{4-8}$.

Allí trabajaron 24 médicos (cirujanos, traumatólogos y clínicos), cuatro odontólogos, dos bioquímicos, 50 enfermeros y 48 suboficiales de sanidad.

El buque además actuó como evacuador de prisioneros de guerra y heridos desde el CIMM hacia el continen- 


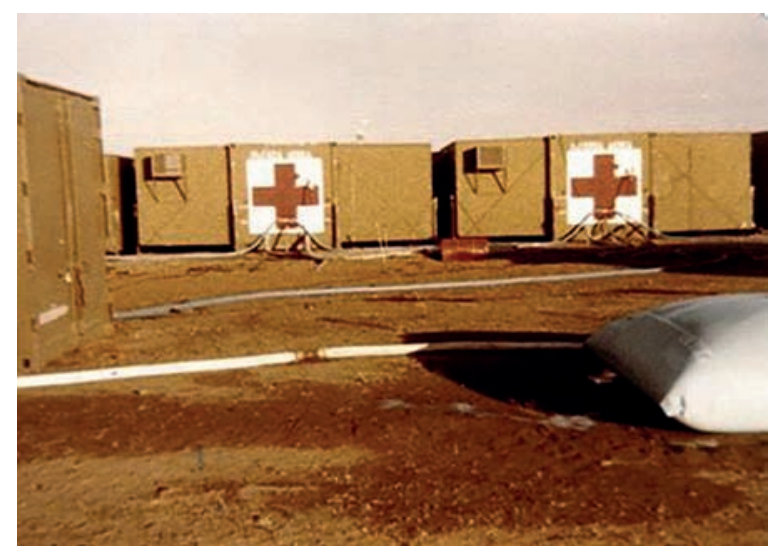

Figura 15. Hospital Reubicable, Comodoro Rivadavia. http://www.3040100.com. arlel-valor-de-una-enfermera-de-la-faa-en-malvinas/ (consultado 07/02/20/8)

te. Al ingreso del paciente se lo dirigía al centro de recepción y clasificación de acuerdo a la vía de llegada respectivamente: aérea o marítima (mediante buques ingleses). Allí eran evaluados por clínico, traumatólogo y cirujano, quienes realizaban el triage e indicaban con lápiz demográfico en las frentes de los pacientes las siglas Q, C o TI (quirófano, internación o terapia intensiva). Los suboficiales de sanidad se encargaban de identificar al paciente y buscar su historia clínica ${ }^{4}$.

Luego se realizaba un segundo examen físico, preponderando la evaluación del SNC, además de realizar exámenes de laboratorio, colocar vías periféricas y despojar al paciente de sus ropas. La preparación prequirúrgica y la toma de placas radiográficas dependían de las lesiones que presentara el paciente.

En el ARA Bahía Paraíso fueron atendidos 231 pacientes, de ellos, 148 fueron heridos en combate, 25 por lesiones fundamentalmente pie de trinchera y 58 por enfermedades comunes, principalmente infecciosas 9

\section{Buque ARA Almirante Irízar (Figura 11)}

Este buque tuvo como tarea la evacuación de heridos. Las tareas de equipamiento comenzaron a principios del mes de mayo en la Base Naval Puerto Belgrano, El 7 de junio el mismo contaba con área de triage, dos quirófanos con sala de esterilización contigua, dos salas de internación general con 105 y 45 camas, terapia intermedia con 4 camas, terapia intensiva con 8 camas, laboratorio de análisis clínicos, sala de yesos, estación de enfermería y pista para el aterrizaje de helicópteros Sea King 231 y 234 (Figura 12), con capacidad de 14 camillas cada uno 4.

El personal arribó desde los Hospitales Navales Pedro Mayo y Puerto Belgrano, y estaba conformado por médicos (intensivista, cirujanos generales (4), traumatólogos (2) terapista, anestesiólogos (2), clínicos (3), cirujano maxilofacial, bioquímico, odontólogo, 11 enfermeros, 6 instrumentadoras quirúrgicas y 36 camilleros.

En este buque se realizaba la estabilización del paciente a través de un triage detalladamente sistematizado, para así devolver al paciente al campo de batalla. El mismo se dividió en dos niveles de atención: el primero destinado a la re-

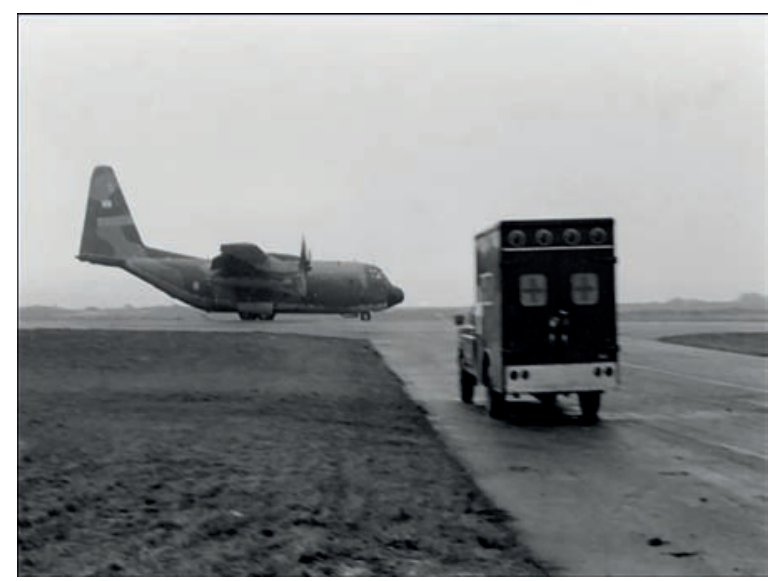

Figura 16. Encuentro entre la ambulancia y el Hércules C-I 30 para evacuar heridos. Ambos no apagaban motores, por si eran sorprendidos por el enemigo y debían actuar rápido.

cepción de los afectados dividiéndolos en atención inmediata, cirugía de urgencia o podían esperar. El objetivo del segundo nivel fue la clasificación de los heridos en:

- Inmediatos (por presencia de lesiones severas en tórax y miembros, o quemaduras de más del 30\%).

- Demorados (si se encontraban clínicamente estables a pesar de presentar lesiones en tórax y miembros, o quemaduras de menos del 30\%).

- Mínimos (si presentaban lesiones no cavitadas, fracturas simples o quemaduras leves).

- Expectantes (tenían pocas probabilidades de sobrevivir).

- Psiquiátricos (especialmente por síndrome postraumático).

Una vez realizado el triage, los tratamientos fueron quirúrgicos o clínicos, y cada uno de ellos se cumplió en forma similar a lo descripto para el buque anterior.

En el caso de que un paciente necesitara transfusiones, el buque contaba con 140 unidades de sangre entera y 150 unidades de plasma, así como con la buena predisposición de donación que manifestó cada uno de los tripulantes.

El buque Almirante Irízar brindó atención médica a 279 pacientes, de los cuales el $50 \%$ fueron heridos por proyectil de artillería; $70 \%$ en miembros superiores y/o inferiores. También se atendieron numerosos pacientes con pie de trinchera. Se registraron solamente dos muertes a bordo, debido a lesiones tóraco-abdominales que no superaron la instancia quirúrgica.

\section{SANIDAD DE LA FUERZA AÉREA ARGENTINA (F.A.A.)}

La recuperación de los heridos no dependió únicamente del trabajo eficiente y profesional del equipo médico, sino también de la rapidez con que los traslados se llevaron a cabo, papel fundamental de la Fuerza Aérea. Para ello el Dr. Mayor Espiniella y el Director General de Sanidad de la F.A, Brigadier Irrgang establecie- 


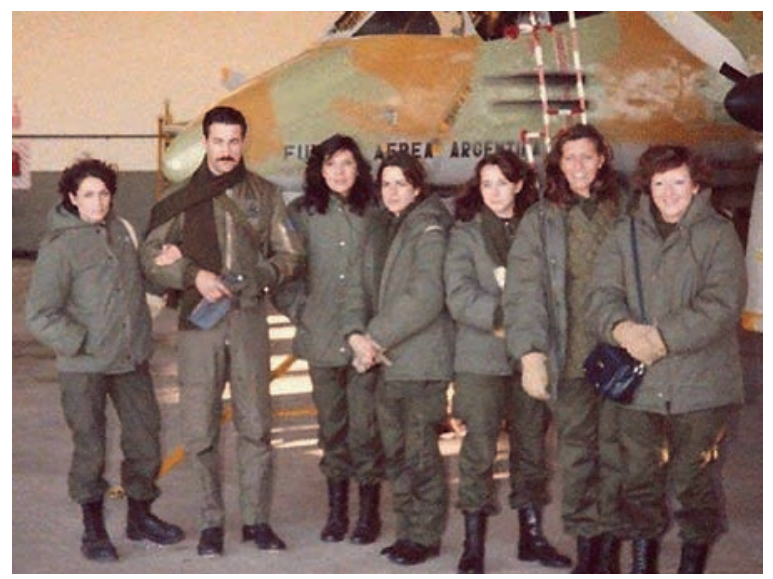

Figura 17. Las instrumentadoras quirúrgicas. http://fdra-malvinas.blogspot.com. ar/2015/02/mujeres-argentinas-en-malvinas.html (consultado 07/02/2018).

ron una logística de evacuación junto con la participación del CIMM y la Fuerza Naval, bajo las normas descriptas en el "Programa para situaciones de emergencia y coordinación del socorro en casos de desastres", de la Organización Panamericana de la Salud de 1977, y adoptada por la Dirección General de Sanidad de la Fuerza Aérea en 1978. Esta última fue la encargada de enviar personal, insumos, víveres, ropa y demás objetos necesarios e imprescindibles para la atención médica objetiva ${ }^{5}$. La F.A estableció dos Bases Aéreas Militares (BAM): Cóndor (Figura 13), en Groose Green en Gran Malvina y Puerto Argentino (Figura 14), en la ciudad homónima en Soledad. Cada una contaba con 30 camillas, equipo completo atención médica enviado el 20 de abril ${ }^{3}$.

En Groose Green el personal se distribuyó en tres lugares estratégicos: puesto de socorro con atención primaria por médico, enfermero y camillero, sala de enfermería y depósito de materiales y BAM Cóndor, desde donde se evacuaba a los heridos al CIMM.

Puerto Argentino contó con todos los niveles de complejidad de atención: puestos de socorro, con dos médicos, tres enfermeros y cuatro soldados camilleros que cubrían turnos de 24 horas, el mismo CIMM y la BAM.

El Hospital Reubicable (HR) (Figura 15) se localizó en Comodoro Rivadavia y funcionó, a partir del 18 de abril, como centro de recepción de bajas provenientes de las islas y depósito de materiales e insumos para los equipos médicos. El personal de atención sanitaria estaba compuesto por dos equipos, cada uno contaba con: cirujanos, traumatólogo, anestesista, clínico, bioquímico y siete enfermeros. El Hospital contaba con salas: de internación general con 35 camas, de cirugía, cuidados intensivos, de rayos, de guardia, de esterilización, laboratorio de análisis y un equipo odontológico ${ }^{3,5}$.

\section{LA EVACUACIÓN, UNTRABAJO EN CONJUNTO}

La logística de evacuación fue establecida por el Dr. Mayor Espiniella y el Dr. Mayor Ceballos, y se organi-

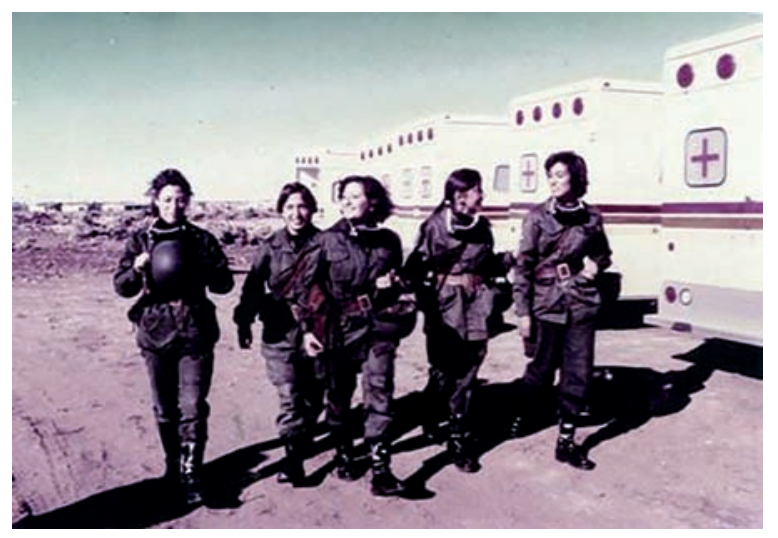

Figura 18. En el Hospital Reubicable: Morales, Bassler, Massito, Reynoso y Maluendez. http://www.3040 I00.com.ar/el-valor-de-una-enfermera-de-la-faa-en-malvinas/ (consultado 07/02/2018)

zó de la siguiente manera desde el punto más cercano al campo de batalla: Puestos de Socorro (PUSO), primer nivel de complejidad de asistencia a heridos transportados por camilleros a pie o ambulancias. Allí se realizaba el primer triage y se estabilizaban allí mismo o eran derivados al CIMM segundo nivel complejidad de atención de heridos. La llegada al CIMM dependía del origen de los heridos, los proveniente de Gran Malvina eran trasportados, primero, a la BAM Cóndor donde eran compensados y luego al CIMM por vía aérea (Figura 16), los provenientes de Soledad eran transportados por ambulancias o soldados camilleros hacia el mencionado centro.

La estructura edilicia del CIMM estuvo destinada a los quirófanos, salas de internación (general, intermedia y terapia intensiva), de rayos, de hemoterapia, cocina, baños para los quemados y lavadero, por lo que el segundo triage se realizó en una carpa adjunta al mismo para luego ser derivados a quirófano o internados para su pronta recuperación, o en su defecto, eran evacuados hacia el tercer nivel de complejidad, los buques hospitales o el Hospital Reubicable en Comodoro Rivadavia.

A partir de los primeros días de junio, el ARA Bahía Paraíso recibió heridos vía aérea, en los aviones sanitarios MERLIN, o vía marítima, a través de los buques hospitales británicos que brindaron atención médica sin distinción de bandera, especialmente el Uganda, a través de los helicópteros PUMA SA-330. En caso que los pacientes necesitasen un tratamiento de mayor complejidad, eran evacuados a Punta Quilla, puerto cercano a Río Gallegos, y luego trasladados, vía aérea, al Hospital Naval Puerto Belgrano. Este buque hospital realizó 4 viajes con el mismo origen y destino con el siguiente detalle

1. Primer viaje; 30 de mayo pasando por Bahía de los Abrigos y Puerto Argentino (Isla Soledad), Red Cross Box para recoger heridos argentinos provenientes del Uganda ( 47 heridos), Bahía Fox (19 heridos) y Puerto Howard (13 heridos) (Isla Gran Malvina). 


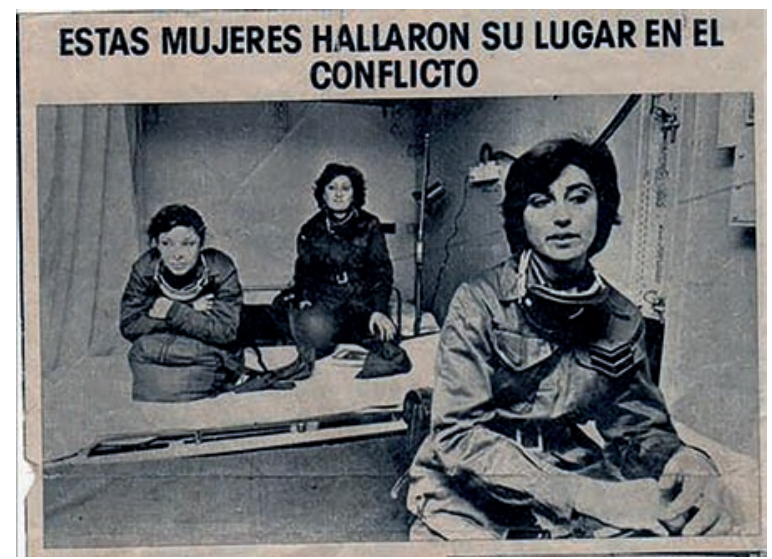

Figura 19. La presencia de las mujeres en Malvinas fue noticia. https://www.infobae.com/2015/03/3//17/9360-la-silenciada-historia-las-veteranas-malvinas/ (consultado 07/02/2018)

2. Segundo viaje; 8 de junio parando en Red Cross Box tanto en la ida como en la vuelta (ningún herido), en Puerto Argentino (10 heridos) y en Bahía Elefante (2 heridos) (Isla Gran Malvina).

3. Tercer viaje; 14 de junio parando en Red Cross Box ( 3 heridos), y en Puerto Argentino.

4. Cuarto viaje; día 20 de junio, parando en Red Cross Box y en Puerto Argentino, para trasladar heridos (44) o bajas directamente hacia Puerto Belgrano.

Se transportaron 609 heridos vía aérea desde el CIMM en Puerto Argentino hacia el Hospital Reubicable, en Comodoro Rivadavia. Cuando fue necesario, se transportaron por vía aérea al Hospital Militar de Campo de Mayo.

\section{REGISTRO NECROLÓGICO}

La búsqueda y recolección de los cadáveres fueron realizadas por el personal dependiente de cada unidad y la evacuación se realizó por vehículos de la unidad, o por helicópteros o ambulancias pertenecientes al CIMM. La evacuación fue realizada por la F.A. hasta el 21 de mayo, fecha en la que las fuerzas inglesas establecen cabecera de playa en San Carlos, impidiendo la llegada de cadáveres al HMPA desde la Isla Gran Malvina, por lo que se dio sepultura allí mismo. Del mismo modo se vio afectada la Isla Soledad a partir del 27 y 28 de mayo, por lo que la sepultura de los cadáveres fue efectuada por manos inglesas.

La identificación y el registro de las bajas se realizaron tanto en los Puestos de Socorro como en el HMPA. La identificación fue una tarea difícil, y eran los mismos soldados quienes determinaban la identidad del cadáver siendo estos compañeros de unidad. Luego un médico certificaba la muerte y la causa de la misma; una vez determinadas ambas, se confeccionaba el certificado de defunción, y se asentaba al caído en el libro del Registro Necrológico del CIMM, el cual determinó un total de 44 cadáveres.

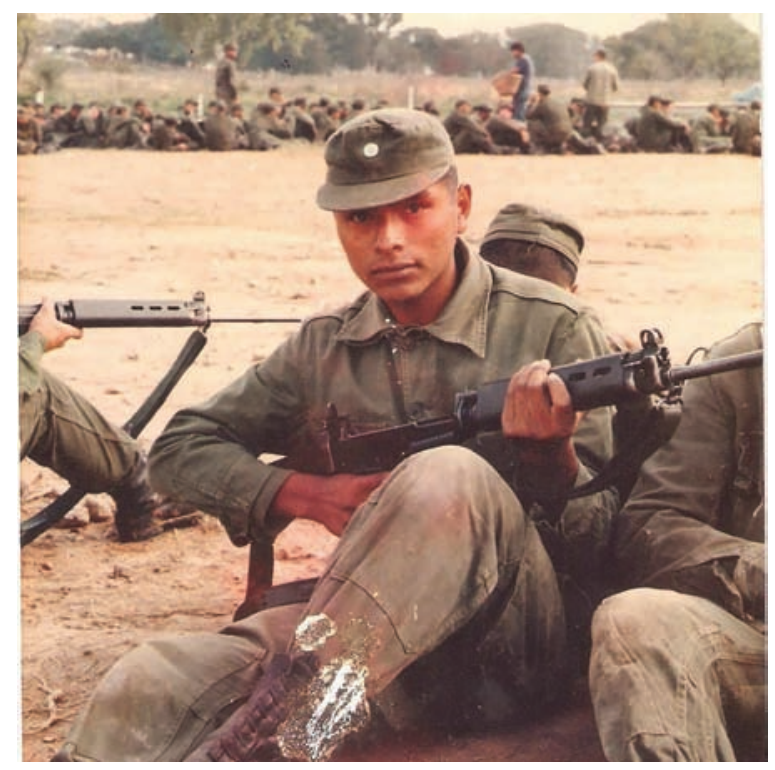

Figura 20. Martín Ignacio Gutiérrez, sosteniendo un fusil a sus 17 años.

La sepultura se llevó a cabo en un predio cercano al HMPA. Los caídos recibían el servicio religioso como así también se le rendían honores militares².

\section{MUJERES, CORONADAS DE GLORIA}

Cuando se hizo público el potencial desenlace bélico entre Argentina y Gran Bretaña, 22 mujeres se inscribieron en la lista de voluntarios del Ejército Argentino, siendo llamadas a entrar en acción entre fines de mayo y principios de junio. Algunas de las 22 mujeres eran instrumentadoras quirúrgicas y fueron destinadas al buque hospital ARA Almirante Irízar donde brindaron tanto su profesionalismo como su empatía por los jóvenes heridos, quienes además de requerir atención médica, requerían de personas que los escucharan, los acogieran y los abrazaran con cálidas palabras, acciones que solo la mujer puede brindar. Sus nombres son María Marta Lemme (Cirugía General), Susana Maza (Cirugía Cardiovascular), Norma Navarro y Cecilia Richieri (Traumatología), María A. Sendes (Cirugía Oftalmológica) y Silvia Barrera (Terapia Intensiva) (Figura 16) ${ }^{7,8}$.

La Cabo Primero Colino fue la primera mujer perteneciente a la Fuerza Aérea que estuvo en las islas, y formó parte de la tripulación sanitaria que acompañó la evacuación de 15 bajas el 13 de mayo desde el CIMM hasta el Hospital Reubicable. Las enfermeras Alicia Mabel Reynoso, Stella Maris Botta, Stella Maris Morales, Gladys Maluendez, María Masitto Anán y Gisella Bassler también pertenecían a la F.A. y ejercieron su labor en el Hospital Reubicable de Comodoro Rivadavia (Figuras 17 y 18)

El resto de las mujeres veteranas de guerra fueron: Olga Cáceres, Cristina Cormack, Maureen Dolan, Graciela Gerónimo, Marta Giménez, Marcia Marchesotti, Mariana Soneira, Silvia Storey y Doris West ${ }^{7}$. 
Así como recordamos a los excombatientes y a los 649 compatriotas que enterrados en suelo malvinense renuevan cada día la soberanía argentina, recordemos también a estas mujeres, que en el medio del fuego y la desesperación, lograron más que cumplir con su objetivo profesional: sedimentar un suelo que para las mujeres aún no era terreno fértil.

\section{PIE DE TRINCHERA: UNA VIEJA PATOLOGÍA REAPARECE EN MALVINAS}

Fue descripta por primera vez en 1916 y fue la patología que prevaleció en los soldados que participaron en la Primera Guerra Mundial; también se diagnosticó durante la II Guerra Mundial y las Guerras de Corea y Vietnam ${ }^{10,11}$. Desde esa época es una afección conoci$\mathrm{da}$, sin embargo en las islas, durante los meses de mayo y junio de 1982, nuestros médicos se enfrentaron por primera vez a esta conocida afección.

Dentro de las afecciones relacionadas al combate de causa médica correspondieron el $96 \%$ de las atendidas en el ARA Bahía Paraíso, al 23\% de las del Hospital Naval Puerto Belgrano, al 16\% de las del ARA Almirante Irízar y al 8\% de las del CIMM.

Es una lesión localizada producida por el frío y que forma parte de las etapas por las que atraviesa el tejido luego al necrosarse por causa del frío. Las etapas, en orden, son las siguientes: síndrome pernio, pie de inmersión, pie de trinchera y congelación. Suele ser de resolución quirúrgica².

El principal factor etiológico es el frío, pero en la producción de este cuadro interactúan otros condicionantes como el tiempo de exposición al frío, el elemento de contacto con el frío y otros factores ambientales como humedad, viento y presión atmosférica. Sumado a factores del huésped, como ser el estado nutricional, presencia de patología vascular periférica, inmovilidad, tolerancia al frío, tabaquismo, consumo de alcohol y el grado militar.

El clima de la zona fue un determinante claro en la aparición de las lesiones. Temperatura entre 2 y $3^{\circ} \mathrm{C}$, humedad entre 80 y $90 \%$, vientos de $300 \mathrm{~km} /$ hora y terreno blando, arcilloso y anegadizo, denominado turba; los combatientes estuvieron inmersos en un medioambiente sumamente favorable para el desarrollo del pie de trinchera. Estos factores contribuyeron a una vasoconstricción arteriolar de los vasos dérmicos dando lugar a la disminución de flujo sanguíneo hacia estructuras más distales, como músculos de las manos y pies y nervios periféricos, a pesar de la formación de shunts arteriovenosos como mecanismo de compensación. Los efectivos se encontraban en constante actividad, por lo que la presión venosa sobre las extremidades fue el doble que lo normal, es decir que la sangre no fluía y generaba estasis capilar, la cual se manifestaba clínicamente con edemas y vesículas. La vaso- constricción contribuyó a este mecanismo, ya que al ser constante generaba estasis y daño endotelial, dando lugar al aumento de la permeabilidad capilar y así a la extravasación del plasma hacia el espacio intercelular; esto último generaba disminución de la presión hidrostática intravascular y concentración de células sanguíneas en el mismo, contribuyendo a la formación de trombos, los cuales, si lograban fluir, producían infarto en la microcirculación distal ${ }^{2,12}$.

Se observó mayor incidencia de pie de trinchera en Gran Malvina que en Soledad, probablemente relacionado a la lejanía del CIMM. Allí se registraron 173 casos de la mencionada afección: 164 correspondieron al Ejército Argentino, 6 a la Fuerza Aérea y 3 a la Marina. Con respecto a las primeras, casi el $90 \%$ eran soldados, la mayoría pertenecientes a los regimientos de infantería, y alrededor del $85 \%$ presentaban lesiones superficiales (eritema, edema, vesículas o ampollas, y necrosis de piel y tejido celular subcutáneo); el 15\% restante presentaba lesiones profundas (necrosis de partes profundas y/o pérdida de tejido), de las cuales solo el $2 \%$ requirió amputaciones mayores (más allá del antepié).

El principal factor que predispuso a la reaparición de esta entidad fue, además de las condiciones climáticas y geográficas del lugar ya descritas, el estado de desnutrición en el cual se encontraban los afectados.

El tratamiento que se empleó consistió en recalentamiento, prohibición de fumar, alimentación adecuada, reposo, control del dolor, antibióticos y actualización de la inmunidad antitetánica ${ }^{2}$. Aquellos que lo requirieron fueron derivados al Hospital Militar de Campo de Mayo y al Hospital Naval Puerto Belgrano donde se contaba con cámara hiperbárica ${ }^{12}$.

\section{MALVINAS, EN PRIMERA PERSONA}

"El 7 de abril egresé como Cabo en Comisión de la Especialidad Intendencia de la Escuela Gral. Lemos, juré la bandera vestido ya para la Guerra; algo sabíamos. Nos fuimos a nuestros hogares para comentarles a nuestras familias que éramos suboficiales del Ejército Argentino en tiempo de guerra. Al otro día fui destinado al Hospital Militar de Comodoro Rivadavia, formando parte de la 9na. Brigada de Regimiento de Infantería, como reemplazos, porque la mayoría éramos menores de edad, yo tenía 17 años, y además por nuestra especialidad. En la madrugada del 9 de abril nos trasladaron a Malvinas, fue todo muy rápido, no hubo tiempo para reaccionar ni decir una palabra. Cuando llegamos a las islas nos destinaron a hacer un campamento de sanidad en el frente de combate y luego a condicionar el Hospital Militar de Puerto Argentino, y ahí trabajamos todos, a pesar de ser suboficiales u oficiales. Mi trabajo fue múltiple: ayudaba en enfer- 
mería, hacía guardias, pintaba las cruces y ayude a construir el helipuerto.

"El primer mes fue tranquilo, trabajamos tranquilos ultimando detalles porque nadie se esperaba lo que todos sabemos. A fines de abril empezaron a bombardear los Harriers, y allí nos dimos cuenta que estábamos en guerra, porque empezaron a llegar heridos, muertos... fue todo un caos: gente quejándose, sufriendo, y nosotros corriendo, pero siempre ocupando nuestros puestos, eso fue lo que nos transmitió el Dr. Ceballos, y así funcionó. Trabajamos toda la noche, yo estuve en la parte de registro necrológico ayudando a identificar a los difuntos, y trabajé junto con un médico y un enfermero. Esa noche no me la voy a olvidar más. "Durante el mes de mayo empezamos a ver casos de neumonías, pies de trinchera, además de los heridos por armas de guerra. Recuerdo que llegaron más profesionales, de primerísimo nivel, porque me asombraba cómo trabajaban en equipo con un único fin: salvar la vida, no solo a nuestros hombres sino también a heridos ingleses.
"El hospital empezó a quedar chico, así que nos mandaron a hacer una morgue con una carpa de campaña. Yo trabajé ahí, trasladando a los muertos en bolsas negras, hasta que nos quedamos sin bolsas y tuvimos que, lamentablemente, acomodarlos unos sobre otros.

"Cuando se acercaba el final, estuve a cargo de la Sala de Terapia Intermedia, hasta que los ingleses bombardearon el pueblo, y tuvimos que correr para evacuar a todos y evitar que caigan prisioneros. A mí me tomaron prisionero junto con otros compañeros y estuve en el Camberra. Fue muy duro, tremendo... pero yo fui por la Patria, todos estábamos orgullosos por ir a defender nuestro país. Estuvimos a punto de ganar, porque fuimos guerreros".

\section{Gutiérrez, Martín Ignacio (Figura 20) \\ Excombatiente de Malvinas}

Agradecimientos a él, padre de la autora principal, por la revisión completa de este manuscrito.

\section{BIBLIOGRAFÍA}

I. Manson E.Tras su manto de neblinas. Buenos Aires. Ed. Fabro. 20I I. Capítulos VI-IX. Páginas 77-196.

2. Ceballos EM. Buroni JR. La Medicina en la Guerra de Malvinas. Buenos Aires. Círculo Militar. 1992. Capítulos II-IV. Páginas 27-119.

3. Fuerza Aérea Argentina - Dirección General de Salud. Memoria: Sanidad de la Fuerza Aérea en el Conflicto del Atlántico Sur (1982). Buenos Aires. 2012. Páginas 9-31.

4. Lo Balbo A. Destino: Atlántico Sur. Parte B: Relatos de Sanidad. Buenos Aires. 2012. Páginas 17-93.

5. Espiniella F.Artículo. La Sanidad de la Fuerza Aérea Argentina en la Guerra de las Islas Malvinas. https://www.escuadronfenix.org. ar/notasycomentarios/Sanidad\%20FAA\%20en\%20Malvinas.html (consultado 0I/02/20|8)
6. Pellicari P, López JA, Parola MAD. Sanidad Naval en Malvinas. Buque Hospital ARA Bahía Paraíso. Boletín del Centro Naval. Nº 833. 20 I 2. Páginas 2I 4-220

7. Pozzio M. La experiencia de las mujeres en Malvinas: de la Sanidad Militar al reconocimiento. Cuadernos de Marte, N 8. 2015. Páginas 129-152

8. http://unw.irizar.org/malvinas-acciones- I.html (consultado 05/02/20 I8).

9. http://www.irizar.org/FAA-Enfermeras.html (consultado 06/02/20।8).

10. Soltau B.Trench feet. RArmy Med Corps 19|6; 26:688-92

I I. Atenstaedt RLTrench foot: the medical response in the firstWorldWar 19|4-19|8.WildernessEnvironMed 2006; 17(4):282-9.

12. Horgan FG,Garay R, Sangiorgi A, Orsi HR. Lesiones por frío. Experiencia con cámara hiperbárica. Pie de trinchera. Boletín del Centro Naval, Nº30. 20 I I. Páginas |80-181. 